\title{
Modelo matemático para el diseño de sistemas de riego tecnificado de baja carga en parcelas pequeñas
}

\author{
E. Vázquez-Fernández y M. Sifuentes-Cabrera \\ Instituto de Ingeniería, UNAM y Fideicomiso de Riesgo Compartido, SAGAR \\ E-mail:ernesvf@servidor.unam.mx
}

(recibido: septiembre de 2000; aceptado: julio de 2001)

\begin{abstract}
Resumen
Se propone un modelo matemático para el diseño de redes de tuberías empleadas en el riego localizado de baja carga $(0.3$ a $1.3 \mathrm{~m})$, donde la emisión del flujo es a través de perforaciones en manguera de polietileno, goteros, difusores o microtubos. El sistema puede ser fijo o móvil. En este último, la manguera con los emisores podrá moverse para regar varias líneas de plantas. El ejemplo que se presenta corresponde a un terreno plano, pero el modelo numérico considera el caso en donde se presenten accidentes topográficos. Se muestran mediciones de campo en tres sistemas móviles diseñados con el modelo numérico para terreno plano. Se concluye que éstas son muy aproximadas a los resultados de gastos y coeficientes de variación obtenidos con el modelo numérico.
\end{abstract}

Descriptores: cabezal, manguera lateral o regante, coeficiente de variación.

\section{Abstract}

A mathematical model for the design of water pipe networks used in on the spot low-head irrigation (0.3 to $1.3 \mathrm{~m}$ ) is proposed.

The output flow is through holes in a polyethylene hose, drippers, diffusors or micropipes. The system can be fixed or mobile. In the latter case the hose with the emmitters could be moved to watwr several rows of plants. The example that is presented corresponds to a flat piece of land, but the numerical model considers the case where the landis not flat, field measurements for three mobile systems designed with the numerical model for flat land are shown. It is concluded that they are flows and coefficients of variation obtained from the numerical model.

Keywords: head, lateral hose for irrigation, coefficient of variation.

\section{Introducción}

El beneficio por la venta del producto cosechado o por el consumo propio que pueden recibir los pequeños agricultores en zonas áridas de escaso crecimiento económico, al emplear sistemas móviles de riego tecnificado en pequeñas parcelas, es muy importante para ellos, por lo que el diseño de dichos sistemas debe estar sustentado en principios hidráulicos básicos para garantizar un buen funcionamiento.

En el riego localizado a través de tuberías de polietileno con cargas de presión altas (mayores de $5 \mathrm{~m}$ ) se emplean, en general, emisores o goteros de diámetro muy pequeño, de 0.4 a $0.7 \mathrm{~mm}$, que son sensibles a la obturación (Rodrigo et al., 1992). En sistemas de baja carga los diámetros pueden ser ligeramente mayores, por ejemplo, Polak et al. (1997) han empleado con éxito sistemas de este tipo para el riego de pequeñas parcelas (del orden de $1000 \mathrm{~m}^{2}$ ), y proponen uno de tipo móvil que reduce los costos a $1 / 4$ del sistema tradicional de alta carga a través de perforaciones directas en la tubería con orificios de $0.7 \mathrm{~mm}$ y cargas de 2,3 y $4 \mathrm{~m}$; los gastos promedios medidos en campo fueron de $6.32,8.53$ y $10.64 \mathrm{l} / \mathrm{h}$, respectivamente. Los laterales o mangueras regantes son de $18.75 \mathrm{~m}$ con 25 emisores a cada $0.75 \mathrm{~m}$.

En el diseño que se propone en este trabajo, las longitudes de los laterales pueden ser más cortas, de 9 a $15 \mathrm{~m}$, con 12 a 36 emisores por lateral, pero hacia ambos lados del cabezal para que la pérdida de carga sea menor a lo largo de 
la tubería, la disminución de la influencia de la temperatura en la variación del gasto y, sobre todo, reducir el costo al moverse los laterales a uno y otro lado. El modelo matemático se adaptó de uno elaborado para calibración de redes de agua potable (Vázquez, 1991) e incluye su descripción a partir de las ecuaciones básicas de pérdida de carga local y de fricción en tuberías, así como de gastos de emisión en goteros o difusores. Los resultados del modelo numérico y la comparación con mediciones de campo muestran la bondad de la aproximación teórica.

\section{Ecuaciones básicas}

El flujo en una manguera lateral o regante de polietileno que tenga emisiones a cada cierta distancia, puede ilustrarse como se muestra en la figura 1 .



Figura 1. Flujo en una manguera lateral o regante con emisores

Una de las fórmulas más empleada para el cálculo de las pérdidas por fricción en tuberías es la de Darcy-Weisbach

$$
h_{j}-h_{k}=\frac{f L_{j k}}{12 \cdot 1 D_{j k}^{5}}\left|Q_{j k}\right| Q_{j k}
$$

donde $h_{j}$ es la carga piezométrica en $\mathrm{m}$, en el punto de emisión j (ver figura 1); $h_{k}$ es la carga piezométrica en m, en el punto de emisión k; fes un coeficiente de fricción, adimensional; $L_{j k}$ es la separación entre emisores en m; 12.1 es una constante que incluye el valor de la aceleración de la gravedad; $D_{j k}$ es el diámetro de la tubería lateral en m; $Q_{\mathrm{jk}}$ es el gasto en $\mathrm{m}^{3} / \mathrm{s}$, que fluye por la tubería entre los emisores j, k.

Para el gasto que fluye entre los puntos $\mathrm{i}, \mathrm{j}$ de la figura 1 , la ecuación 1 puede expresarse de manera semejante como

$$
h_{i}-h_{j}=\frac{f L_{i j}}{12.1 D_{i j}^{5}} Q_{i j} Q_{i j}
$$

La ecuación para pérdida de carga local por la inserción de un gotero en el punto $\mathrm{j}$ de la manguera lateral puede expresarse en función del gasto aguas arriba como

$$
\Delta h_{j}=\frac{\alpha}{12.1 D_{i j}^{4}} Q_{i j} Q_{i j}
$$

donde $\Delta \mathrm{h}_{\mathrm{j}}$ es la pérdida de carga local en el punto de emisión j, en m; $\alpha$ es un coeficiente adimensional de pérdida local por la inserción del emisor en la manguera.

Así, la ecuación de pérdida de carga total entre los puntos $i, j$ se escribe

$$
h_{i}-h_{j}=\frac{f L_{i j}}{12.1 D_{i j}{ }^{5}}\left|Q_{i j}\right| Q_{i j}+\frac{\alpha}{12.1 D_{i j}{ }^{4}} Q_{i j} Q_{i j}
$$

La otra ecuación básica es la del emisor, que se acostumbra escribir como

$$
Q_{j}=C_{j}\left(h_{j}-z_{j}\right)^{b}
$$

donde $Q_{j}$ es el gasto de emisión en el punto j, en $1 / h$; $C_{j}$ es una constante del gotero o difusor empleado como emisor; $h_{\mathrm{j}}$ es la carga piezométrica en $\mathrm{m}$; $z_{\mathrm{j}}$ es la carga de posición o cota del terreno en $\mathrm{m}$; b es un exponente menor de 1 .

\section{Modelo matemático}

a) Ecuación fundamental en la manguera lateral o regante

De la ecuación 4 se puede obtener en forma implícita el gasto $Q_{i j}$ como

$$
Q_{i j}=\frac{h_{i}-h_{j}}{B_{i j}\left|Q_{i j}\right|}
$$

donde $B_{i j}$ es una constante que vale

$$
B_{i j}=\frac{f L_{i j}}{12 \cdot 1 D_{i j}{ }^{5}}+\frac{\alpha}{12 \cdot 1 D_{i j}{ }^{4}}
$$

La ecuación de continuidad en cualquier punto j de emisión, que en este inciso se llamará nodo, así como las conexiones entre las mangueras lateral y distribuidora donde no hay emisión (por ejemplo, el punto i de la figura 1), puede expresarse de manera implícita como

$$
-\frac{h_{i}-h_{j}}{B_{i j}\left|Q_{i j}\right|}+\frac{h_{j}-h_{k}}{B_{j k}\left|Q_{j k}\right|}+\frac{C_{j}}{(1000)(3600)}\left(h_{j}-z_{j}\right)^{b}=0
$$


$\mathrm{Al}$ ordenar esta ecuación se obtiene

$$
\begin{aligned}
-\frac{1}{B_{i j}\left|Q_{i j}\right|} h_{i}+ & {\left[\frac{1}{B_{i j}\left|Q_{i j}\right|}+\frac{1}{B_{j k}\left|Q_{j k}\right|} h_{j}-\frac{1}{B_{j k}\left|Q_{j k}\right|} h_{k}=\right.} \\
& -\frac{C_{j}}{(1000)(3600)}\left(h_{j}-z_{j}\right)^{b}
\end{aligned}
$$

En esta ecuación lineal se ha dividido la constante C entre (1000) (3600) para convertir el gasto de la ecuación 5 a $\mathrm{m}^{3} / \mathrm{s}$.

b) Ecuación de continuidad en el extremo final de la manguera lateral

Supóngase que los puntos de emisión j, k son los nodos extremos de la manguera lateral en una red abierta; entonces, la ecuación de continuidad 9 en el nodo extremo $\mathrm{k}$ se convierte en

$$
-\frac{1}{B_{j k}\left|Q_{j k}\right|} h_{j}+\frac{1}{B_{j k}\left|Q_{j k}\right|} h_{k}=-\frac{C_{k}}{(1000)(3600)}\left(h_{k}-z_{k}\right)^{b}
$$

c) Ecuación de continuidad en un nodo de la manguera distribuidora

La figura 2 muestra en planta una conexión de la manguera distribuidora con dos nodos opuestos al i, separados, en la misma manguera, una distancia $\mathrm{L}_{\mathrm{mi}}=\mathrm{L}_{\mathrm{in}} \mathrm{Y}$ la conexión con el nodo j en la manguera lateral.

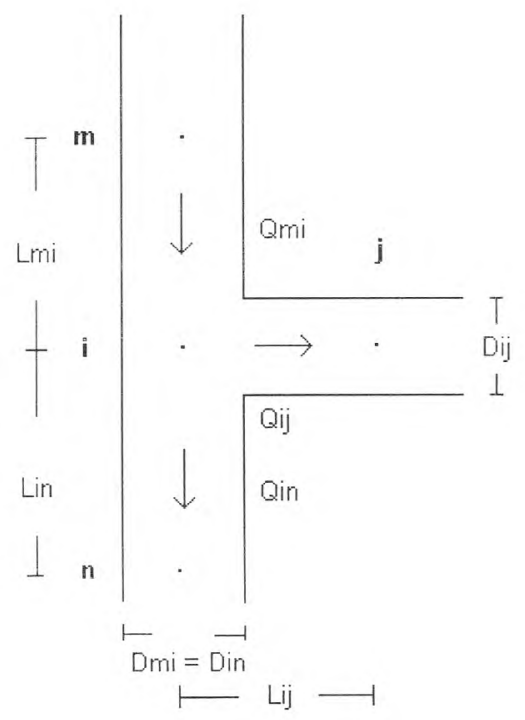

Figura 2. Conexión de la manguera distribuidora con un lateral
Si no hay emisión en el nodo i, la ecuación de continuidad queda

$$
\begin{gathered}
-\frac{1}{B_{m i}\left|Q_{m i}\right|} h_{m}+\left[\frac{1}{B_{m i}\left|Q_{m i}\right|}+\frac{1}{B_{i j}\left|Q_{i j}\right|}+\frac{1}{B_{i n}\left|Q_{i n}\right|}\right] h_{i}- \\
\frac{1}{B_{i j}\left|Q_{i j}\right|} h_{j}-\frac{1}{B_{i n}\left|Q_{i n}\right|} h_{n}=0
\end{gathered}
$$

En esta ecuación las constantes $\mathrm{B}_{\mathrm{mi}}, \mathrm{B}_{\mathrm{in}}$ son

$$
B_{m i}=\frac{f L_{m i}}{12.1 D_{m i}{ }^{5}} ; B_{i n}=\frac{f L_{i n}}{12.1 D_{i n}^{5}}
$$

Nótese que ya no se incluye el término donde interviene $\alpha$ porque en la manguera distribuidora no hay emisores conectados a ella.

d) Ecuación de continuidad en un nodo extremo de la manguera distribuidora

Si ahora el nodo i fuera el extremo de la manguera distribuidora y no existiera el nodo n, la ecuación de continuidad en el primer nodo se escribe

$$
-\frac{1}{B_{m i}\left|Q_{m i}\right|} h_{m}+\left[\frac{1}{B_{m i}\left|Q_{m i}\right|}+\frac{1}{B_{i j}\left|Q_{i j}\right|} h_{i}-\frac{1}{B_{i j}\left|Q_{i j}\right|} h_{i}=0\right.
$$

e) Ecuación de continuidad en un nodo contiguo a una condición de frontera

Supóngase ahora que el nodo $\mathrm{m}$ sobre la manguera distribuidora es una condición de frontera con carga piezométrica conocida y constante, esto es, que sea el cabezal o tanque de alimentación a la red de mangueras. La ecuación de continuidad en el nodo i contiguo es

$$
\begin{gathered}
{\left[\frac{1}{B_{m i}\left|Q_{m i}\right|}+\frac{1}{B_{i j}\left|Q_{i j}\right|}+\frac{1}{B_{i n}\left|Q_{i n}\right|} \mid h_{i}-\frac{1}{B_{i j}\left|Q_{i j}\right|} h_{j}-\right.} \\
\frac{1}{B_{i n}\left|Q_{i n}\right|} h_{n}=\frac{1}{B_{m i}\left|Q_{m i}\right|} h_{m}
\end{gathered}
$$

Las ecuaciones $9,10,11,12,13$ cubren todos los casos para las ecuaciones de continuidad en los nodos de una red abierta como la de la figura 3 , donde se observa el cabezal en el centro del área de riego que alimenta a dos mangueras distribuidoras y éstas, a su vez, a doce laterales cada una. 


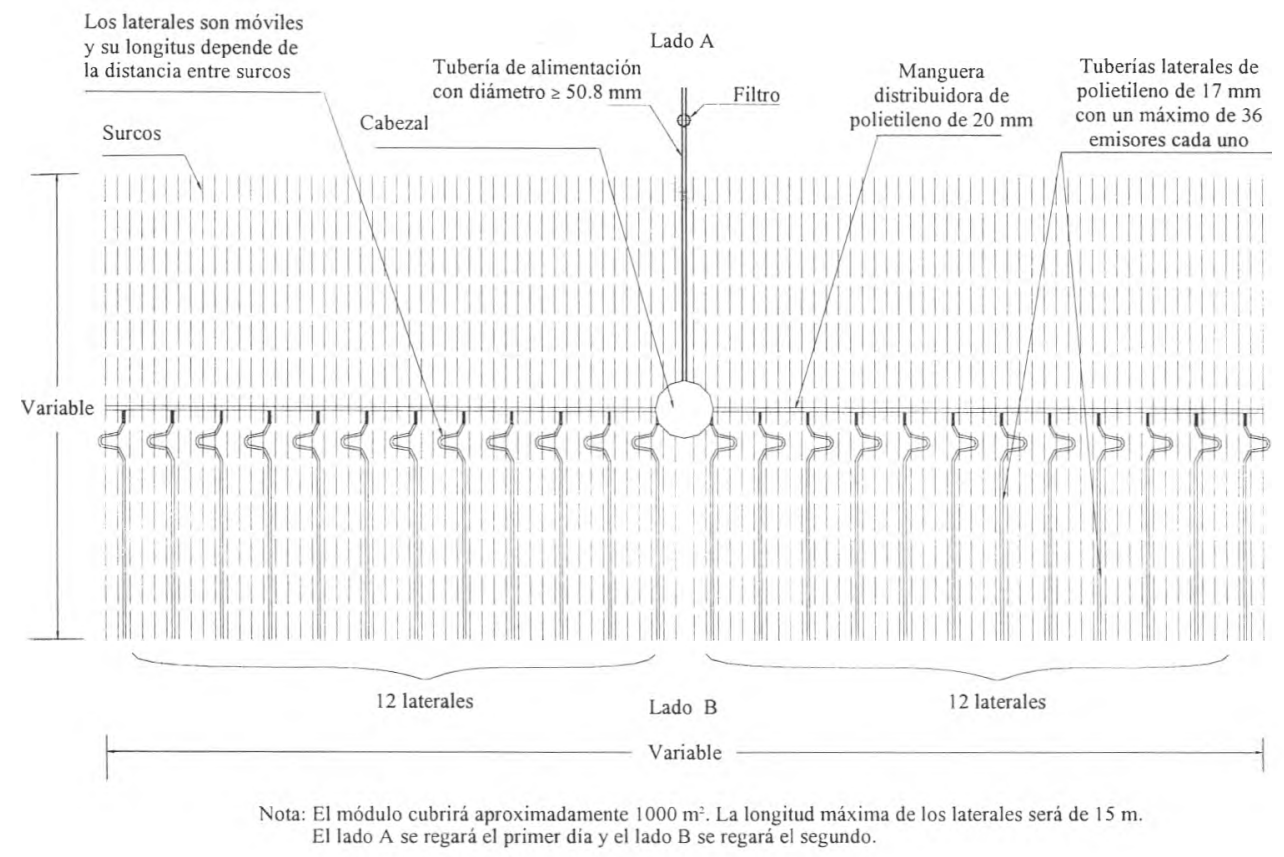

Figura 3. Sistema móvil con manguera perforada, goteros o difusores

Al plantearse el sistema de ecuaciones en los nodos de la red, sean de emisión o no, se establece un sistema de ecuaciones lineales igual al número total de nodos, donde las incógnitas son las cargas piezométricas.

Como el sistema es implícito es necesario conocer los gastos iniciales en cada tramo de las mangueras, los cuales se determinan a partir de unas cargas piezométricas supuestas. Estas deben tomar valores decrecientes, según el sentido del flujo, a partir de la carga conocida en el cabezal. Estos gastos se calculan de manera general, con la ecuación 1 escrita en la forma

$$
Q_{i j}=\frac{h_{i}-h_{j}}{\left|h_{i}-h_{j}\right|} \sqrt{\frac{\frac{h_{i}-h_{j} \mid}{f L_{i j}}}{12.1 D_{i j}^{5}}}
$$

En esta ecuación deberán emplearse las distancias y diámetros correspondientes (subíndices ij), según se trate de un lateral o una manguera distribuidora entre los nodos $i, j$. Además, el signo del gasto queda determinado por la diferencia $h_{i}-h_{j}$.

El proceso de solución es iterativo al emplear en el cálculo siguiente los valores obtenidos en la solución anterior, hasta que las cargas piezométricas se repitan dentro de una cierta tolerancia, por ejemplo $0.001 \mathrm{~m}$. En un sistema de ecuaciones lineales de 300 nodos, al resolver con el método exacto de Crout (Hildebrand, 1973), el número mínimo de iteraciones es 6 (el máximo puede ser 10), por lo que es necesario elaborar el programa en un lenguaje que realice los cálculos de manera rápida, por ejemplo, el FORTRAN.

Una vez conocidas las cargas piezométricas para cada nodo, dentro del programa se determinan los gastos de emisión con la ecuación 5 , así como el promedio de los gastos $(\overline{\mathrm{Q}})$ en toda la red, la desviación estándar $\left(\sigma_{Q}\right)$ y, con ellos, el coeficiente de variación $\left(\mathrm{Cv}=\sigma_{\mathrm{Q}} / \overline{\mathrm{Q}}\right)$, que es el principal parámetro estadístico para decidir sobre la bondad del diseño, ya que, por ejemplo, el gasto está relacionado con el tiempo de riego y puede modificarse de acuerdo con el tipo de suelo.

Si el terreno es plano $\left(z_{\mathrm{j}}=z_{\mathrm{k}}=0\right)$, las ecuaciones 9 y 10 se simplifican como sigue

$$
\begin{gathered}
-\frac{1}{B_{i j}\left|Q_{i j}\right|} h i+\left[\frac{1}{B_{i j}\left|Q_{i j}\right|}+\frac{1}{B_{j k}\left|Q_{j k}\right|}+\frac{C_{j}}{(1000)(3600)\left(h_{j}\right)_{a}^{1-b}}\right] h_{j}- \\
\frac{1}{B_{j k}\left|Q_{j k}\right|} h_{k}=0 \\
-\frac{1}{B_{j k}\left|Q_{j k}\right|} h j+\left[\frac{1}{B_{j k}\left|Q_{j k}\right|}+\frac{C_{k}}{(1000)(3600)\left(h_{k}\right)_{a}^{1-b}}\right] h_{k}=0
\end{gathered}
$$

En estas ecuaciones se incluye un artificio de cálculo que consiste en dividir las constantes $C_{j}$ y $C_{k}$ entre $\left(h_{j}\right)_{a}^{1-b}$ y $\left(h_{k}\right)_{a}^{1-b}$, donde $\left(h_{j}\right)_{a},\left(h_{k}\right)_{a}$ son los valores de las cargas 
piezométricas en cualesquier nodos j, k en la iteración anterior a la de cálculo en la solución del sistema de ecuaciones, de manera que en la última iteración los valores de las cargas en los nodos con emisión son iguales o muy aproximados, aceptándose que dichas cargas quedan elevadas al exponente b, como en la ecuación 5 . Este artificio permite una convergencia más rápida en la solución del sistema de ecuaciones porque todas ellas quedarán igualadas a cero, excepto las que se apliquen en algún nodo i adyacente al cabezal (como en la ecuación 13).

\section{Diseño de un sistema móvil para regar un terreno plano de $972 \mathrm{~m}^{2}$}

Sea la red abierta de tuberías del sistema que se muestra en la figura 3, con 12 laterales de manguera de polietileno (baja densidad resistente al alto intemperismo) de $17 \mathrm{~mm}$ de diámetro exterior y $13.4 \mathrm{~mm}$ de diámetro interior. Los laterales se encuentran conectados a una manguera distribuidora, con las mismas características de la anterior, pero de $20 \mathrm{~mm}$ de diámetro exterior y de $16.5 \mathrm{~mm}$ de diámetro interior. Esta última se conecta por dos lados a un cabezal que tiene una carga de $1 \mathrm{~m}$. Los emisores conectados a las mangueras laterales tienen una separación de $0.75 \mathrm{~m}$, y consisten en difusores de $0.89 \mathrm{~mm}$ de diámetro cuya ecuación cargas-gastos (ver ecuación 5) obtenida en el laboratorio tiene una constante $\mathrm{C}=7.07 \mathrm{y}$ un exponente $b=0.57$. La separación entre laterales es de $2.25 \mathrm{~m}(3 * 0.75)$, esto es, cada lateral regará a tres surcos o líneas de plantas; además de este movimiento en paralelo en el lado A, los laterales se moverán hacia el lado B opuesto para cubrir un área total de $972 \mathrm{~m}^{2}$ $(2 * 24 * 3 * 0.75 * 12 * 0.75)$. La longitud de los laterales será de $11 \mathrm{~m}(12 * 0.75+2)$ para alcanzar a cubrir las tres líneas de plantas por lado. Si sólo se emplea en el cálculo $1 / 4$ del área total, es decir, la mitad de los laterales que se instalarán, el número de ecuaciones por resolver es de 156 $(12 * 12+12)$, siendo 144 nodos con emisión y 12 nodos de conexión con la manguera distribuidora.

De acuerdo con las ecuaciones presentadas en el modelo matemático, el esquema del sistema de ecuaciones lineales tiene el siguiente orden:
1) nodo contiguo al cabezal en la manguera distribuidora = ecuación 13
2) nodocrucero entre la manguera distribuidora y un lateral = ecuación 11 , etc.
3) nodo extremo en la manguera distribuidora $=$ ecuación 12
4) nodo al principio de un lateral conectado a la manguera distribuidora $=$ ecuación 15 , etc.
5) nodo intermedio en un lateral = ecuación 15 , etc.
6) nodo extremo en un lateral = ecuación 16, etc.

Por ejemplo, según la numeración de nodos de la figura 4, el esquema del sistema de ecuaciones para las primeras 13 ecuaciones (nodos 2 a 14) es el de la tabla 1.

Al emplear los coeficientes promedio, $f=0.03$ y $\alpha=$ 0.2 , dados por Bagarello et al. $(1995,1997)$, el modelo numérico proporciona un gasto promedio de $4.6 \mathrm{l} / \mathrm{h}$ y un coeficiente de variación de 0.14 , por lo que el diseño se considera aceptable. Rodrigo et al. (1992) indican los intervalos siguientes para el CV

$\begin{aligned} \quad C V \leq 0.05 & \text { Excelentes } \\ 0.05<C V \leq 0.07 & \text { Normales } \\ 0.07<C V \leq 0.11 & \text { Marginales } \\ 0.11<C V \leq 0.15 & \text { Deficientes } \\ 0.15<C V & \text { Inaceptables }\end{aligned}$



Figura 4. Numeración de los 15 primeros nodos de la red

\section{Mediciones de campo}

El sistema móvil se instaló en tres localidades de la Comarca Lagunera, México; en dos de ellas con 24 laterales de 12 emisores cada uno y en otra con 20 laterales de 14 emisores cada uno. En las tablas 2, 3 y 4 se presentan las mediciones de gastos realizadas a un solo lado del cabezal (mitad de laterales instalados), tanto en los emisores extremos de cada lateral, como en el ubicado al centro de los mismos, es decir, no se midieron todos los emisores, pero se considera que estos aforos son comparables con el diseño teórico, ya que se midieron emisores representativos del mismo número de laterales. En las tablas se anotan las cargas en el cabezal durante la medición, los gastos medios, los CV y las diferencias con respecto al obtenido en el diseño teórico $(\triangle \mathrm{CV})$. 
Tabla 1. Esquema del sistema de ecuaciones para las primeras 15 ecuaciones

\begin{tabular}{|c|c|c|c|c|c|c|c|c|c|c|c|c|c|c|c|}
\hline Nodo/Carga & $\mathrm{h}_{2}$ & $\mathrm{~h}_{3}$ & $\mathrm{~h}_{4}$ & $\mathrm{~h}_{5}$ & $\mathrm{~h}_{6}$ & $\mathrm{~h}_{7}$ & $\mathrm{~h}_{8}$ & $\mathrm{~h}_{9}$ & $h_{10}$ & $\mathrm{~h}_{11}$ & $\mathrm{~h}_{12}$ & $\mathrm{~h}_{13}$ & $\mathrm{~h}_{14}$ & $\mathrm{~h}_{15}$ & $\begin{array}{l}\text { Término } \\
\text { Independiente }\end{array}$ \\
\hline 2 & $\mathrm{X}$ & $\mathrm{X}$ & & & & & & & & & & & & $x$ & $\mathrm{x}$ \\
\hline 3 & $\mathrm{X}$ & $\mathrm{X}$ & $\mathrm{X}$ & & & & & & & & & & & & \\
\hline 4 & & $\mathrm{X}$ & $\mathrm{X}$ & $\mathrm{X}$ & & & & & & & & & & & \\
\hline 5 & & & $\mathrm{X}$ & 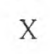 & X & & & & & & & & & & \\
\hline 6 & & & & $\mathrm{X}$ & $\mathrm{X}$ & $\mathrm{X}$ & & & & & & & & & \\
\hline 7 & & & & & $\mathrm{X}$ & $\mathrm{X}$ & $\mathrm{X}$ & & & & & & & & \\
\hline 8 & & & & & & $\mathrm{X}$ & $x$ & $\mathrm{X}$ & & & & & & & \\
\hline 9 & & & & & & & $\mathrm{X}$ & $\mathrm{X}$ & $\mathrm{X}$ & & & & & & \\
\hline 10 & & & & & & & & $\mathrm{X}$ & $\mathrm{X}$ & $\mathrm{X}$ & & & & & \\
\hline 11 & & & & & & & & & $\mathrm{x}$ & $\mathrm{x}$ & $\mathrm{X}$ & & & & \\
\hline 12 & & & & & & & & & & $\mathrm{x}$ & $\mathrm{X}$ & $\mathrm{x}$ & & & \\
\hline 13 & & & & & & & & & & & $\mathrm{X}$ & $\mathrm{x}$ & $\mathrm{x}$ & & \\
\hline 14 & & & & & & & & & & & & $\mathrm{X}$ & $\mathrm{x}$ & & \\
\hline
\end{tabular}

Tabla 2. Medición de gastos en "Viesca". Carga en el cabezal $=0.65 \mathrm{~m} \overline{\mathrm{Q}}=2.44 \mathrm{l} / \mathrm{h} ; \mathrm{CV}=0.208$;

$\Delta / C V=0.14-0.208=-0.068$

\begin{tabular}{ccc}
\hline No. de lateral & Ubicación del emisor en el lateral & Gasto $[1 / \mathrm{h}]$ \\
\hline \multirow{2}{*}{2} & Principio & 2.11 \\
& Medio & 1.88 \\
2 & Final & 2.34 \\
& Principio & 3.94 \\
& Medio & 2.84 \\
3 & Final & 3.28 \\
& Principio & 1.80 \\
& Medio & 1.78 \\
4 & Final & 1.71 \\
& Principio & 2.39 \\
& Medio & 2.03 \\
5 & Final & 2.11 \\
& Principio & 3.00 \\
& Medio & 2.81 \\
6 & Final & 2.37 \\
& Principio & 2.89 \\
& Medio & 2.53 \\
& Final & 2.61 \\
& Principio & 3.21 \\
& Medio & 2.77 \\
& Final & 2.49 \\
& Principio & 3.08 \\
& Medio & 2.71 \\
& Final & 2.57 \\
\hline
\end{tabular}

continuación...

\begin{tabular}{ccc}
\hline \hline No. de lateral & Ubicación del emisor en el lateral & Gasto $[1 / \mathrm{h}]$ \\
\hline \multirow{4}{*}{9} & Principio & 2.97 \\
& Medio & 3.08 \\
& Final & 3.65 \\
10 & Principio & 3.91 \\
& Medio & 3.08 \\
& Final & 3.39 \\
11 & Principio & 3.35 \\
& Medio & 2.60 \\
& Final & 3.06 \\
12 & Principio & 3.04 \\
& Medio & 3.28 \\
\hline \hline
\end{tabular}

Tabla 3. Medición de gastos en "La Ventana". Carga en el cabezal $=0.70 \mathrm{~m} \overline{\mathrm{Q}}=3.68 \mathrm{l} / \mathrm{h} ; \mathrm{CV}=0.106$; $\Delta C V=0.14-0.106=0.034$

\begin{tabular}{ccc}
\hline \hline No. de lateral & Ubicación del emisor en el lateral & Gasto $[1 / \mathrm{h}]$ \\
\hline \multirow{2}{*}{1} & Principio & 3.39 \\
& Medio & 3.86 \\
2 & Final & 3.68 \\
& Principio & 3.41 \\
& Medio & 3.26 \\
& Final & 3.09 \\
\hline
\end{tabular}


continuación...

\begin{tabular}{|c|c|c|}
\hline No. de lateral & Ubicación del emisor en el lateral & Gasto $[1 / \mathrm{h}]$ \\
\hline & Prncipio & 3.68 \\
\hline \multirow[t]{3}{*}{3} & Medio & 3.48 \\
\hline & Final & 3.86 \\
\hline & Principio & 3.88 \\
\hline \multirow[t]{3}{*}{4} & Medio & 3.55 \\
\hline & Final & 3.17 \\
\hline & Principio & 3.35 \\
\hline \multirow[t]{3}{*}{5} & Medio & 3.33 \\
\hline & Final & 3.48 \\
\hline & Principio & 3.56 \\
\hline \multirow[t]{3}{*}{6} & Medio & 3.51 \\
\hline & Final & 2.76 \\
\hline & Principio & 3.42 \\
\hline \multirow[t]{3}{*}{7} & Medio & 3.79 \\
\hline & Final & 3.77 \\
\hline & Principio & 3.78 \\
\hline \multirow[t]{3}{*}{8} & Medio & 3.97 \\
\hline & Final & 4.00 \\
\hline & Principio & 4.04 \\
\hline \multirow[t]{3}{*}{9} & medio & 4.16 \\
\hline & Final & 3.96 \\
\hline & Principio & 4.50 \\
\hline \multirow[t]{3}{*}{10} & Medio & 4.65 \\
\hline & Final & 4.49 \\
\hline & Principio & 3.60 \\
\hline \multirow[t]{2}{*}{11} & Medio & 3.60 \\
\hline & Final & 3.60 \\
\hline
\end{tabular}

\section{Discusión}

Obsérvese que el CV del sistema instalado en "Alto de Palomillo" (tabla 4) tiene un CV menor al límite de inaceptable y su diferencia $(\triangle \mathrm{CV})$ con respecto al valor teórico de 0.14 es mínima. En el sistema instalado en "La Ventana" (tabla 3) el CV obtenido en campo (0.106) es inferior al valor teórico, por lo que en estos dos casos puede decirse que los resultados del modelo numérico, para el CV, son muy aproximados a los medidos en campo. En cuanto al sistema instalado en "Viesca" (tabla 2), se tiene un CV que se clasifica como inaceptable; sin embargo, dados los resultados de la cosecha obtenida en esta última localidad, donde se sembró ajo (Allium sativum) con una producción de 7 Ton/ha, ligeramente mayor a la máxima de la región (Sandoval, 1999), con un ahorro de agua del 50\% en comparación con una parcela testigo de $224 \mathrm{~m}^{2}$ regada por gravedad, el valor del CV $=0.208$ obtenido durante un
Tabla 4. Medición de gastos en "Alto de Palomillo". Carga en el cabezal $=0.74 \mathrm{~m} \overline{\mathrm{Q}}=3.80 \mathrm{l} / \mathrm{h}: \mathrm{CV}=0.145$; $\Delta \mathrm{CV}=0.14-0.145=-0.005$

\begin{tabular}{|c|c|c|}
\hline No. de lateral & Ubicación del emisor en el lateral & Gasto $[1 / \mathrm{h}]$ \\
\hline & Principio & 3.47 \\
\hline \multirow[t]{3}{*}{1} & Medio & 4.66 \\
\hline & Final & 4.05 \\
\hline & Principio & 4.59 \\
\hline \multirow[t]{3}{*}{2} & Medio & 4.34 \\
\hline & Final & 4.60 \\
\hline & Principio & 4.64 \\
\hline \multirow[t]{3}{*}{3} & Medio & 4.52 \\
\hline & Final & 3.80 \\
\hline & Principio & 4.05 \\
\hline \multirow[t]{3}{*}{4} & Medio & 3.16 \\
\hline & Final & 3.09 \\
\hline & Principio & 3.74 \\
\hline \multirow[t]{3}{*}{5} & Medio & 3.72 \\
\hline & Final & 3.95 \\
\hline & Principio & 3.91 \\
\hline \multirow[t]{3}{*}{6} & Medio & 3.47 \\
\hline & Final & 3.48 \\
\hline & Principio & 3.67 \\
\hline \multirow[t]{3}{*}{7} & Medio & 3.82 \\
\hline & Final & 3.44 \\
\hline & Principio & 3.94 \\
\hline \multirow[t]{3}{*}{8} & Medio & 3.02 \\
\hline & Final & 3.69 \\
\hline & Principio & 4.13 \\
\hline \multirow[t]{3}{*}{9} & Medio & 3.84 \\
\hline & Final & 2.18 \\
\hline & Principio & 4.12 \\
\hline \multirow[t]{2}{*}{10} & Medio & 3.44 \\
\hline & Final & 3.42 \\
\hline
\end{tabular}

día de medición indica que el límite de inaceptabilidad debería ser mayor para el riego de parcelas pequeñas. Es decir, en éstas puede disminuirse la uniformidad sin existir merma en el rendimiento, suponiendo que las actividades agronómicas se cumplen con oportunidad.

Por otra parte, aunque hay diferencias entre la carga teórica en el cabezal ( $1 \mathrm{~m}$ ) y las medidas en campo, el CV no se modifica de manera significativa porque las desviaciones de los gastos con respecto al valor medio se conservan constantes, es por eso que las diferencias en los CV del diseño teórico y las mediciones de campo son mínimas. En cambio, si se redujera por ejemplo, la separación entre emisores o se ampliara el diámetro de la 
manguera distribuidora, conservando el mismo tipo de emisor, se experimentaría una disminución notable en el CV; así, las variables más importante en el diseño son la separación entre emisores, los diámetros de las mangueras, el tipo de emisor y, en menor medida (en ese orden), el coeficiente de pérdida de carga por fricción y el de pérdida local. No se menciona la temperatura, ya que por la corta longitud de los laterales, las altas temperaturas de la región donde se instalaron los sistemas, en ocasiones de $40^{\circ} \mathrm{C}$, no influyeron de manera negativa en la variación de los gastos; sin embargo, en un estudio posterior podría verificarse esta afirmación.

Las simulaciones con el modelo numérico para las cargas de operación en el cabezal, $0.33 \mathrm{~m}, 0.66 \mathrm{~m}$ y $0.70 \mathrm{~m}$, proporcionan valores de CV iguales a $0.13,0.14$ y 0.14 , respectivamente, esto es, la diferencia con respecto al valor obtenido para $1 \mathrm{~m}$ es nula en los dos últimos casos y muy pequeña (0.01) para el primero.

Hay que destacar que las opciones para el diseño con el modelo numérico pueden ser diferentes en cuanto a variables, como la separación entre plantas o emisores, diámetros de mangueras, goteros, número de laterales por mover en sistemas móviles, disposición de las mangueras en sistemas fijos y áreas de riego, por lo que el diseño aquí presentado es solo un caso entre una gran variedad.

\section{Conclusiones}

De acuerdo con las pequeñas diferencias entre los CV calculados con el modelo matemático y los obtenidos en campo (ver valores $\Delta C V$ en tablas 2,3 y 4 ), se concluye que el modelo presentado es muy confiable para el diseño de sistemas de riego tecnificado en parcelas pequeñas, siempre y cuando se conozca con suficiente aproximación la ecuación cargas-gastos de los emisores.

No obstante, las diferencias en las cargas de diseño y de operación en el cabezal, por lo mencionado anteriormente, puede afirmarse que el cumplimiento de la carga de diseño no es relevante. Los tiempos de riego pueden adecuarse a los gastos promedio que se obtengan en campo. Lo importante es que la carga sea suficiente para garantizar la emisión del flujo en los puntos más alejados de la red de tuberías. Véase en las tablas 2, 3 y 4 que las cargas de operación fueron menores a la de $1 \mathrm{~m}$ empleada en el diseño.

\section{Agradecimientos}

Al Fideicomiso de Riesgo Compartido (FIRCO) en la Comarca Lagunera por su apoyo en las mediciones de campo, a los ingenieros Rafael de la Cruz, Pedro López y Laura Vélez por su valiosa colaboración en los estudios de laboratorio, al M.I. Víctor Franco y al Dr. Jesús Gracia por la revisión crítica del escrito.

\section{Referencias}

Bagarello V., Ferro V., Provenzano G. y Pumo D. (1995). Experimental Study on Flow-Resistance Law for Small-Diameter Plastic Pipes. Journal of Irrigation and Drainage Engineering, Vol. 121, No. 5.

Bagarello V., Ferro V., Provenzano G.y Pumo D. (1997). Evaluating Pressure Losses in Drip-Irrigation Lines. Journal of Irrigation and Drainage Engineering, Vol. 123, No. 1.

Hildebrand F.B. (1973). Métodos de la Matemática Avanzada. Editorial Universitaria de Buenos Aires, apéndice.

Polak P., Nanes B. y Adhikari D. (1997). A Low Cost Drip Irrigation System for Small Farmers in Developing Countries. Journal of the American Water Resources Association, Vol. 33, No. 1.

Rodrígo J., Hernández J.M., Pérez A. y González J.F. (1992). Riego Localizado. Centro Nacional de Tecnología de Regadios, España.

Sandoval G.R. (1999). El cultivo del ajo (Allium sativum L.) en Viesca, Coahuila. Memoria de experiencia profesional para obtener el título de ingeniero agrónomo. Universidad Autónoma Agraria "Antonio Narro", Unidad Laguna, p. 11.

Vázquez E. (1991). Calibración de un modelo matemático estático en sistemas de agua. Universidad y Ciencia, Vol. 8, No. 16, Universidad Juárez Autónoma de Tabasco. 


\section{Semblanza de los autores}

Ernesto Vázquez-Fernández. Egresado de la UNAM, ha sido profesor de la Universidad Juárez Autónoma de Tabasco y de la DEPFI, UNAM. Asimismo, se ha desempeñado como especialista en ingeniería hidráulica en los sectores público y privado. Actualmente, labora en el Instituto de Ingeniería, UNAM.

Miguel Sifuentes-Cabrera. Ingeniero Agrónomo egresado de la Escuela Superior de Agricultura y Zootecnia de la Universidad Juárez del Estado de Durango, con la especialidad en el uso y conservación del agua (Irrigación). Ha tomado varios cursos de capacitación, entre ellos, Métodos de riego, Uso de plásticos en la agricultura, Formulación y evaluación de proyectos y Diseño de sistemas de riego a presión. Ha sido Supervisor Técnico y Jefe de Estudios y Proyectos en el programa de Capacitación y Empleo Cooperativo para el Fomento de Recursos Naturales en Zonas Marginadas (COPLAMAR). Asimismo, Coordinador de Técnicos y Revisor de los expedientes técnicos de ferti-irrigación en la Gerencia Estatal Comarca Lagunera del Fideicomiso de Riesgo Compartido (FIRCO). Actualmente tiene a su cargo el diseño, instalación y supervisión de los proyectos de riego tecnificado en pequeñas parcelas en esta última dependencia. 